\title{
Improved Air Traffic Management through fuzzy approach on Conflict Detection and Resolution using Smart Antenna
}

\author{
Puthanial. $\mathrm{M}^{1}$, Dr P. C. Kishore Raja ${ }^{2}$ \\ Saveetha School of Engineering, Saveetha University, Chennai, India
}

\begin{abstract}
Air traffic management is responsible for smooth air traffic. Although it is done by lot of computations with human interactions, the system will be at its best if there are reduced computations and less flight delay time. This system deals with an introduction of fuzzy rules at the point where they are conflict detection. So with the set of rules implying on the detection, resolution can be made on the basis of fuzzy rules. Ensuring the separation and other criteria in lieu with other aircrafts, the resolution is computed. The computations are reduced either by spatial separation or temporal separation. In the present system there is hardly any conflict detection or decision support. The conflict detection computes and derives with the resolution which reaches the ATC (Air traffic control). This paper describes how fuzzy ideas are used with the conflict detection and how the decision support is made with reduced calculations and improved air traffic flow with a reduced delay
\end{abstract}

Index Terms: ATC, conflict detection, Optimization, AOC, Sensors, Smart Antenna

\section{Introduction}

Most of the decision support tools for controlling the aircraft is developed by conflict detection algorithms. Without designated routes aircraft cannot proceed. These routes are designed based on algorithms and visual Communication. These algorithms are developed to ensure the aircraft safety and also follow the minimum separation rules; providing proper Navigation, communication and security to the public.

Existing methods follow many models. But the most common one is the Euclidian distance between the aircrafts which concentrates on maintaining the minimum separation distance. In our country there is a there is a lack of sophisticated or automated controllers resulting in limited usage of controllers.

The computations are procured in three different algorithms like $\mathrm{X}^{2}, \mathrm{X} \log 2 \mathrm{X}$ and in least case it is $\mathrm{X}$. There are various factors which influence the aircraft commute Where in some may be anticipated and some may not be. In the existing design comparison of visual approach and cockpit display of traffic information enhanced flight rules gives general reference to the speed management, where $\mathrm{X}$ being the number of aircraft

Information from the AOC, weather, and data marked, and set of instructions are all the parts of aircraft influence.

\section{Back Ground}

Traffic flow management and ATC (Air traffic control) helps the ATM in facing all the traffic challenges and ensure a smooth along with the Air traffic control is his work involves the improved scheduling method designed with a collaborative Flow management. The problem focused in our project is Airline Schedule recovery in Airspace sectors capacity limited conditions due to convective weather conditions.

The objective of this paper is to re plan the traffic flows around the network of traffic constraints and to increase the quantity of the automation supported planning benefits by introducing the fuzzy rules at the conflict detection improving the decision support. This model is embedded in dynamic simulation environment; this model involves the current and future operations of airline behaviors based on optimization formulation. This paper includes the comparison of the different effectiveness of Automation in replanning. Two methods of comparisons are followed here; one is comparing the replanning schedules of flight departure using ground delays, flight cancellations and pre departure re-routing using coded departure routes (CDR). Second method is an idealized convective weather disruption data is produced to generate significant airspace capacity outages for many hours to verify the difference between current and more advanced planning techniques.

This model specializes only the departures from single airport, but this methodology is general to apply to NAS-wide airline schedules .Additionally this method addresses both the airport and airspace capacities. This paper shows the significant delay reduction benefits can be achieved if more automation is available to support the Traffic flow management at different weather conditions.

ATC provides services to aircraft in flight between airports as well. Pilots fly under one of two sets of rules for separation they are VFR and IFR, Visual flight rules (VFR) are a set of regulations which allow a pilot 
to operate an aircraft in weather conditions generally clear enough to allow the pilot to see where the aircraft is going. Specifically, the weather must be better than basic VFR weather minimums, as specified in the rules of the relevant aviation authority. If the weather is worse than VFR minimums, pilots are required to use instrument flight rules(IFR).At busy airports maximum efficiency and minimum delay occur when visual approaches are being conducted by pilots using visual separation from traffic. This responsibility of the pilot increases the flexibility of the controllers in traffic flow management. We can improve this efficiency in low weather conditions where the pilots continue the same separation tasks by referring the Cockpit Display Of traffic Information (CDTI) procedure used by ATC. In this application, the flight crew is authorized to use the CDTI. The cockpit display of traffic information (CDTI) enhanced flight rules (CEFR) application is an extension of the current visual separation during the period of visual obscuration, the pilot will use CDTI to maintain awareness of the relative position and speed of other traffic. After passing through the visibility restricting condition, and prior to landing, the flight crew will use the CDTI to assist CDTI will also be used to initially detect, monitor, and reacquire the traffic to follow more effectively.

This study examines the instrument approach application with particular attention to the effect of CDTI location and size responses. Speed management was used to adjust spacing to a pilot-determined value. The results indicate that pilots are willing and able to perform the CEFR procedure irrespective of the CDTI sizes or locations which can be tested in simulation along with the fuzzy set of rules modifying the existing data.

\section{Human Factors Implications Of These Changes}

The information that is actually required for decision making in the air traffic control system. A Great deal of data determines what is an effective or ineffective decision in this complex distributed system. Indicates that there is also a great deal of variability in the system, some of it under control of human managers and operators, but much of it not controllable (weather, etc.). The aviation system is highly dynamic and data describing the system changes constantly, sometimes in unanticipated events, in particular, is something unanticipated ways. Coping with computers do not do well. This fact emphasizes the system's dependence on human operators who can cope with such variance, regardless of whether pilots or controllers are making the decisions.

Model figure to predict temperature and humidity

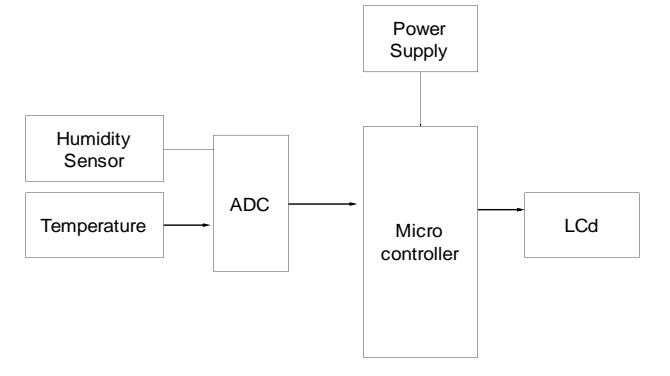

Thus the block explains the prediction of temperature and humidity. The main reason for aircraft delay is the weather data, airport conditions and data from TMU. Effective management of ATM information will be absolutely critical to success of a future system based on free flight concepts.

\section{Conclusion}

The main theme of this paper is that in some situations, decision-making requires reasoning about uncertainty and consideration of data that is not easily communicated to a computer. Both of these factors imply that humans will continue to have the responsibility for maintaining full situation awareness and for integrating all of the relevant information to make decisions. Computer systems with capabilities such as conflict detection and resolution will simply be some of many sources of information that the people in the system must consider.

The second theme is how to predict the uncontrollable problems like the weather and prediction of temperature and humidity which may reduce delay time of aircraft to a larger extent.

\section{References}

[1] B. Sridhar, G.B Chatterji, "Computationally efficient Conflict detection methods for Air Traffic Management", 1997 Proceedings of the American Control Conference.

[2] Philip J. Smith, Charles E. Billings, David D. woods, c. Elaine McCoy, "Human Factors issues in Air Traffic Management”

[3] Schwab, R.W., Haraldsdottir, A., Kostiuk, P., Simpson R.W., Wright R., Wiseman R., Chen S., and Ware B., 2000, A Preliminary 
Design Process for Airspace Systems, Initial Assessment - Chicago Case Study. Report Prepared for NASA Ames Research Center, Department of Transportation. Report No. VNTSC-DTS20-PDP-001.

[4] Boeing Commercial Airplanes Current Market Outlook, www.boeing.com/ commercial/cmo

[5] Zhang, Y., Menendez, M., Hansen, M., Analysis of de-peaking strategies implemented by American Airlines: causes and effects, presentedat the 83rd annual meeting of Transportation Research Board, January,2004.

[6] Lovell, D., et al, 2007, Calibrating Aggregate Models of Flight Delays and Cancellation Probabilities At Individual Airports, 7th USA/Europe ATM R\&D Seminar, Barcelona, Spain.

[7] Smith, D., and L. Sherry, 2009, Decision Support Tool for Predicting Ground Delay Programs (GDP) and Airport Delays From Weather Forecast Data, Annual Transportation Research Board (TRB) Conference, Washington, DC

[8] Klein, A., R. Jehlen, S. Kavoussi, D. Hickman,D. Simenauer, M. Phaneuf, and T. MacPhail, 2008,"Quantification of Predicted Impact of Weather on Air Traffic and Comparison With Actual Impact", 13th Conference on Aviation, Range and Aerospace Meteorology, AMS, New Orleans, LA.

\section{Summary}

1. Puthanial. M, Associate, Professor, Electronics and Communication Engineering Department, Saveetha School of Engineering,

Saveetha University, Chennai .puthanial@gmail.com

2. P.C Kishore Raja, Professor and Head, Electronics and Communication Engineering Department, Saveetha School of Engineering, Saveetha University, Chennai , pckishoreraja@gmail.com

Puthanial. M is pursuing her PhD in Wireless communication related work in the area of smart antennas under the guidance of Dr. P. C. Kishore Raja, Professor and Head, Department of Electronics and communication who completed his Doctorate from Anna university. My thanks to the UG students Narala Rohit Reddy, Md Juned Kalam and Darga Reddy for working closely on this article. 